A Comparison of the Sensitivity of Four Indirect Evaluation Measures to Evaluative

Information

Yoav Bar-Anan

Ben-Gurion University of the Negev, Beer-Sheva, Israel.

Brian A. Nosek

University of Virginia and Center for Open Science, USA.

Indirect evaluation measures are used as a dependent measure to assess the impact of experimental interventions on shifting pre-existing attitudes or creating new attitudes. In four experiments (total $N=13,894$ ), we compared the sensitivity of four indirect evaluation measures to evaluative information about two novel targets. Evaluative sensitivity was strongest for the Implicit Association Test (IAT). Other measures were more similar in their sensitivity, but the pattern, from stronger to the weakest was the Affect Misattribution Procedure (AMP), the Sorting Paired Features (SPF), and then Evaluative Priming task (EPT). To the extent that these findings are generalizable to related research applications, these results suggest that the measures differ in their research efficiency (power). For example, to achieve $80 \%$ power to detect the evaluative learning effect in the present studies, direct self-report would require 10 participants, the IAT 28 participants, the AMP 57, the SPF 79, and the EPT 131.

Keywords: Attitudes; Evaluation; Indirect Measures; Implicit Measures; Automatic Evaluation

Author's note: Correspondence should be addressed to: Yoav Bar-Anan, Department of Psychology, Ben-Gurion University in the Negev Be'er Sheva, Israel. E-mail: baranany@bgu.ac.il. This project was supported by grants from the Israeli Science Foundation [779/16] to Y. B.-A, and from Project Implicit Inc. and the United States - Israel Binational Science Foundation [2013214] to Y. B.-A and B.A.N.. B.A.N. is an officer of Project Implicit Inc., a nonprofit organization that provided financial and technical support to this project, and includes in its mission "To develop and deliver methods for investigating and applying phenomena of implicit social cognition, including especially phenomena of implicit bias based on age, race, gender or other factors." The authors declare that there are no potential conflicts of interest with respect to the research, authorship, and/or publication of this article. 


\section{A Comparison of the Sensitivity of Four Indirect Evaluation Measures to Evaluative Information}

Evaluation of objects as positive or negative is a ubiquitous human behavior.

People judge whether they like or dislike politicians, job candidates, potential partners for friendship or romance, food, appliances, and scientific manuscripts. The study of the factors that determine likes and dislike is central to social psychology (Ajzen \& Fishbein, 1980; Albarracin, Johnson, \& Zanna, 2014; Anderson, 1965; Asch, 1946; Hovland, Janis, \& Kelly, 1953; Petty \& Cacioppo, 1986; Walther \& Langer, 2008). Experimental investigations of evaluation require an evaluation measure as a dependent variable. Selfreports of evaluation are common, partly because they are relatively easy to implement and partly because participants' explicit, deliberate evaluation is the variable of interest. Alternative measures are indirect in that participants do not need to consciously reflect on their evaluation for assessment (Nosek \& Greenwald, 2009). Indirect measures of evaluation are used, partly because participants might otherwise report the evaluation they want to appear to have (Jones \& Sigall, 1971; Leary \& Kowalski, 1990), or because they may not be aware of all aspects of their evaluation. It is common to assume that indirect measures of evaluation reflect automatic evaluation whereas most self-report measures capture deliberate evaluation (De Houwer, 2006; Fazio \& Olson, 2003; Nosek, Hawkins, \& Frazier, 2011).

To improve the knowledge about the various indirect evaluation measures, research on their psychometric is essential. Such research examines the reliability and validity of the measures (e.g., Banse, Seise, \& Zerbes, 2001; Egloff \& Schmukle, 2002; Spruyt, Hermans, De Houwer, Vandekerckhove, \& Eelen, 2007), investigates what 
psychological processes influence those measures (Blaison, Imhoff, Hühnel, Hess, \& Banse, 2012; Conrey, Sherman, Gawronski, Hugenberg, \& Groom, 2005; Gawronski, Deutsch, LeBel, \& Peters, 2008), and what modifications could improve them (Nosek, Greenwald, \& Banaji, 2005; Payne, Cheng, Govorun, \& Stewart, 2005). Most psychometric evidence comes from studies focused on a single indirect measure. However, in reality, researchers must weigh the evidence comparatively to decide which indirect measures are most appropriate for their research. Comparing separate studies of each measure is fraught with procedural, design, and sampling confounds. There are some comparative studies of indirect measures, but all so far have been correlational designs (e.g., Bar-Anan \& Nosek, 2014; Bar-Anan, Nosek, \& Vianello, 2008; Bosson, Swann, \& Pennebaker, 2000; Cunningham, Preacher, \& Banaji, 2001; De Houwer \& De Bruycker, 2007; Karpinski \& Steinman, 2006; Krause, Back, Egloff, \& Schmukle, 2011; Payne, Govorun, \& Arbuckle, 2008; Ranganath, Smith, \& Nosek, 2008; Rudolph, Schröder-Abé, Schütz, Gregg \& Sedikides, 2008). For example, Bar-Anan and Nosek (2014) measured attitudes about race, politics, and the self with seven indirect measures, and compared the measures on reliability and relations to direct evaluation measures, and to the other indirect measures.

In the present research, we compared the validity of indirect evaluation measures experimentally. We manipulated evaluative information about two novel target objects, and then measured the automatic evaluation of those objects. We compared four indirect measures: The Implicit Association Test (IAT; Greenwald, McGhee, \& Schwartz, 1998; Nosek, Greenwald, \& Banaji, 2007), the Evaluative Priming Task (EPT; Fazio, Sanbonmatsu, Powell, \& Kardes, 1986; Klauer, \& Musch, 2003), the Affect 
Misattribution Procedure (AMP; Payne et al., 2005; Payne \& Lundberg, 2014), and the Sorting Paired-Features task (SPF; Bar-Anan, Nosek, \& Vianello, 2009). IAT, EPT and the AMP are the three most commonly used indirect evaluation measures (Nosek, Hawkins, \& Farzier, 2012). We included the SPF because, for some research purposes, especially in the domain of evaluative learning, it is a promising alternative to the IAT because it measures all the task conditions in the same block, and it has previously outperformed EPT (Bar-Anan et al., 2008; Bar-Anan \& Nosek, 2014).

In a previous correlational comparison, the ranking of the four measures by their psychometric qualities was IAT, SPF, AMP, EPT (Bar-Anan \& Nosek, 2014). However, in a fellow-up study with a larger number of AMP trials, measuring only race attitudes, the AMP was similar to the IAT in most criteria, and showed the strongest relation with self-reported attitudes (Bar-Anan \& Nosek, 2013). In a study that included the IAT, AMP, and EPT (Payne, Govorun, \& Arbuckle, 2008) in a measurement of attitudes toward alcohol, the AMP was the only measure with reliable relations with six reported drinking behaviors, whereas the IAT and EPT were each related reliably only to one reported behavior. However, the sample size was too small to reach reliable conclusions about comparative performance. Finally, in a direct comparison of the SPF and EPT for measurement of political attitudes, the SPF showed better reliability and validity than the EPT (Bar-Anan, Nosek, \& Vianello, 2008).

The experimental induction of attitudes toward novel target objects for comparative purposes in the present studies has three main strengths. First, an experimental design provides better control of the variance of the evaluations, and is not reliant on pre-existing variation in evaluations. We provided evaluation information that 
ranged from one extreme (an extremely more positive information about object $\mathrm{A}$ than about object B) to another (an extremely more positive information about object B than about object A), with equal likelihood for any extremity. This distribution is ideal for assessing measurement sensitivity.

Second, in correlational studies, the evidence depends on the reliability and validity of the criterion measures. For instance, Bar-Anan and Nosek (2014) found that the AMP was comparatively weakly related with other indirect measures. That could indicate a weakness of the AMP or that other indirect measures are all confounded by a non-evaluative construct that inflated their inter-correlations. In the present study, we examined how much variance each indirect measure shared with the evaluative information that we provided experimentally providing an independent assessment of measurement quality and sensitivity.

A third strength of the present research is that it is uniquely relevant for research on evaluative learning. Research on impression formation (Ratliff \& Nosek, 2010; Rydell, McConnell, Strain, Claypool, \& Hugenberg, 2007), persuasion (Horcajo, Briñol, \& Petty, 2010; Smith, De Houwer, \& Nosek, 2013), and other forms of evaluative learning (Olson \& Fazio, 2001; Stahl \& Unkelbach, 2009) often uses novel objects as stimuli and indirect measures as the dependent variable. Previous comparative studies of indirect measures addressed concepts that participants have known for days, weeks, and years. Knowledge and evaluation of such concepts is different from knowledge formed based on very recent information. For example, recently formed memories have not been fully consolidated (McGaugh, 2004; Stickgold \& Walker, 2005). Therefore, newly formed attitudes might depend on unique retrieval and judgment processes. Perhaps 
indirect measures of evaluation vary in their relative sensitivity to different retrieval processes. If that is the case, previous correlational studies are of less relevance than the present research to the reliability and validity of indirect evaluation measures in the context of evaluative learning research.

\section{Overview of Experiments}

We used the same procedure in all four experiments. Participants read eight behaviors, four positive and four negative. Four behaviors were attributed to a man named Kevin, and four attributed to a man named Brian. We varied between participants the number of positive and negative behaviors attributed to each man. Theory and empirical evidence suggest that just like deliberate evaluation, automatic evaluation has a straightforward reaction to such information: more positive behaviors cause more automatic liking (Bar-Anan \& Nosek, 2012; Ratliff \& Nosek, 2010; Rydell et al., 2007; Nosek, 2005; Smith et al., 2013). That manipulation is expected to cause clear effects on automatic evaluation regardless of the measure.

We considered inducing automatic evaluation that is distinct from deliberate evaluation because researchers often use indirect measures to tap a construct distinct from deliberate evaluation. However, there is currently no well-replicated method to experimentally induce automatic evaluation that is distinct from deliberate evaluation. In recent research that demonstrates this problem, Moran, Bar-Anan, and Nosek (in press) used an evaluative learning procedure that had a discrepant effect on indirect and direct evaluation measures (Gregg, Seibt, \& Banaji, 2006; Peters \& Gawronski, 2011). In three pairs of studies, each pair used a different indirect measure. Each measure showed different results, and only one measure showed evidence of discrepancy from deliberate 
evaluation. In the present research, although we provided straightforward evaluative information about the targets, for exploration, we still examined whether the indirect measures captured any effect not captured by the deliberate evaluation.

In each of the present experiments, after providing the evaluative information, we measured participants' attitudes toward Kevin and Brian, using one indirect measure, and a direct self-report measure. We compared all four measures in Experiment 1. As replications with a few methodological improvements, we compared the AMP to the IAT in Experiment 2, the SPF to EPT in Experiment 3, and all four measures in Experiment $4^{1}$.

\section{Method}

\section{Participants}

Participants were volunteers at the Project Implicit research website (Nosek, 2005), who registered for research and were randomly assigned to the study from a pool of available studies. In Experiments 1-3, we planned to run about 1,000 participants for each indirect measure in each study to avoid exhausting the shared resource of participants in Project Implicit (https://implicit.harvard.edu/). In Experiments 1-3, power was about $80 \%$ for detecting a correlation difference between .4 and .5 , but only $65 \%$ to detect a difference between .15 and .25 . In Experiment 4, we planned to recruit at least 6000 participants (around 1,500 participants for each measure), to achieve $90 \%$ power to detect a correlation difference between .2 and .3 .

\footnotetext{
${ }^{1}$ We report all data exclusions, manipulations, and measures, and how we determined sample sizes. Decisions to stop collecting data did not depend on the obtained results. Materials, data and links to experience the studies are in https://osf.io/sn9jp/ .
} 
The total number of participants who responded to all the self-report questions and completed the indirect measure was 4,041, 2,145, 1,949, and 6,406 in Experiments 14 , respectively $\left(M_{\text {age }}=34.88,39.03,36.24,35.37 ; S D_{\text {age }}=13.96,16.79,15.26,14.56 ; N\right.$ women $=2,621,1,395,1,267,3,885)^{2}$. Of those, the analyses did not include participants whose performance in the indirect measure suggested lack of ability or motivation to perform the task properly. The exclusion rules were those recommended in previous work with the present data source, for the IAT (Greenwald, Nosek, \& Banaji, 2003), SPF (Bar-Anan \& Nosek, 2014), and EPT (Bar-Anan, 2010; Bar-Anan \& Nosek, 2014; BarAnan, 2016), and the rules recommended based on various participant populations for the AMP (Payne \& Lundberg, 2014). Specifically, the analyses did not include participants who responded faster than $300 \mathrm{~ms}$ in more than $10 \%$ of the IAT trials (exclusion rates: 7/1,043, 3/1,073, 21/1,653 in Experiments 1, 2, and 4), or the SPF trials (13/976, 30/957, 37/1,454 in Experiments 1, 3, and 4), participants who had less than 40\% success-rate in EPT (5/1013, 7/992, 11/1,579 in Experiments 1, 3, and 4), and participants who used only one of the two responses throughout the AMP, or reported having any knowledge of Chinese (108/1006, 120/1,067, 199/1,639 in Experiments 1, 2, and 4). In total, we included in the analyses 3,902, 2,017, 1,912, and 6,063 participants in Experiments 1-4, respectively.

\footnotetext{
2 7,993 participants started Experiment 1, but some data of 1,389 participants was lost for technical problems in the data recording, and 2,564 participants did not finish the study (a drop-out rate of 39\%, which is a typical dropout rate in Project Implicit's volunteer-based participant pool). In Experiment 2-4, the dropout rates were $1,346 / 3,491,1,310 / 3,259,2,849 / 9,234$, respectively.
} 


\section{Procedure}

The experiments were administrated over the Internet using PIPE (Version 0.3; Zlotnick, Dzik, \& Bar-Anan, 2015). Participants who previously registered for the Project Implicit participant pool logged in voluntarily and were randomly assigned to complete this study from a pool of available studies. Once assigned to any of the four experiments, they could not be assigned to any of the four experiments on future visits.

The consent form stated that studies at Project Implicit examine attitudes, preferences, and memory. Participants observed the learning task, completed a questionnaire about their evaluation of the two target men, a questionnaire about their evaluation of each behavior that was presented in the learning task (removed from Experiment 4), and an indirect measure of evaluation toward the two targets. The behavior rating questionnaire appeared before the learning task or at the end of the experiment (randomized between participants). The direct and indirect evaluation measures always appeared after the learning task, in random order. Participants were randomly assigned to complete one indirect measure.

The instructions before the learning task were: In the next task, you will get to know two men. We will tell you a few facts about each of these men. Later, we will ask you a few questions about what you think of them. The learning task had eight trials. On each trial, the man's image, his name, and a behavior he performed were displayed together for 5 seconds, followed by a 750ms blank screen. The trials alternated from one man to the other, for a total of four trials per man. For each participant, four positive and four negative behaviors were randomly selected from a pool of 23 positive and 23 negative behaviors. The five between-subjects randomly-assigned conditions were a ratio 
of positive to negative behaviors attributed to Brian of $0: 4,1: 3,2: 2,3: 1$, and 4:0, always mirrored by the ratio of positive-to-negative behaviors attributed to Kevin (4:0, 3:1, 2:2, 1:3, and 0:4). All procedures are available for self-administration at $\underline{\mathrm{osf} . \mathrm{io} / \mathrm{j} \mathrm{xpb} 8}$ to review details of the methodology.

\section{Measures}

Self-reported evaluation of the behaviors. For each behavior, the participants answered the question How positive or negative is this behavior? on a 9-point scale with the options 'Extremely positive', 'Very positive', 'Moderately positive', 'Slightly positive', 'Neutral', 'Slightly negative', 'Moderately negative', 'Very negative', and 'Extremely negative'. The eight questions were presented one at a time, in a random order, with no mention that the men performed them (approximately half of the participants completed this questionnaire before being presented with the men). We used those ratings to create a corpus of behavior ratings (see Table S4 in the web supplement).

Self-reported evaluation of the men. In Experiments 2-4, participants answered questions in the format of "How trustworthy is Kevin?", for the traits friendly, trustworthy, and likable, on a 9-point scale ranging from 1 - Extremely unfriendly to 9 Extremely friendly. In Experiment 1, as a part of a methodological investigation of question formats, each participant saw the questions in one of six formats (e.g., the scale responses appeared horizontally or vertically; one at a time in separate pages or all in the same page), all with identical or very similar wording and five of them with 9-point response scales (a sixth used visual analog scale that displayed only the most extreme labels on each size of the scale, and was divided to 201 responses). This methodological investigation is not discussed further. 
Affect Misattribution Procedure. Each trial in the AMP displayed stimuli in the following sequence: A photograph of one of the two targets appeared for $100 \mathrm{ms,}$ followed by a blank screen for $100 \mathrm{~ms}$, followed by a Chinese pictograph for $100 \mathrm{ms,}$ followed by a pattern mask of black-and-white noise that appeared until participants responded. Upon presentation of the mask, participants indicated if the Chinese pictograph is more pleasant or more unpleasant than the average Chinese pictograph using two response keys on the keyboard signifying pleasant and unpleasant. Following Payne et al. (2005), the instructions informed participants the pictures appearing before the Chinese pictographs may bias responses and that they should not let the pictures influence their judgments. The task started with three example trials and then participants completed three blocks of 40 trials, each with 20 primes for each man.

Evaluative Priming Task. In Experiments 1 and 3, each trial in the EPT started with a prime (i.e., a photograph of Brian or Kevin) displayed for 200 milliseconds; then a positive or negative target word appeared until participant categorized it as positive or negative using one of two response keys on the keyboard. If the response was incorrect, a red "X" appeared for 750 milliseconds. Last, a blank screen appeared for an inter-trial interval (ITI) randomly selected from a range of $300 \mathrm{~ms}$ to $900 \mathrm{~ms}$. In comparison to the other measures, there is less consensus about the procedural parameters of the EPT. However, inspection of recent literature found only minor differences. In Experiments 1 and 3 we used parameters from our previous work (Bar-Anan, 2009; Bar-Anan \& Nosek, 2014; Moran, Bar-Anan, \& Nosek, in press). In Experiment 4, we used parameters from research by Bertram Gawronski, who has published many recent evaluative learning papers using the EPT (specifically, we used parameters from Gawronski, Gast, \& De 
Houwer, 2015). The modifications relative to the procedure in the previous experiments were that the trial started with a 500ms display of a fixation stimulus before the prime, the error feedback appeared for $1500 \mathrm{~ms}$, and the ITI was fixed to $500 \mathrm{~ms}$.

The rationale behind the EPT is that liked primes facilitate the classification of positive targets, and impede the classification of negative targets; whereas disliked primes facilitate the classification of negative targets, and impede the categorization of positive targets. Therefore, the automatic preference for one prime over another is inferred by comparing the facilitation in responses to positive targets versus negative targets after one prime versus the other prime. In Experiments 1 and 4, the EPT consisted of three blocks of 60 trials (15 trials for each prime-target combination). In Experiment 3 , each block consisted of 40 trials (10 trials for each prime-target combination). Participants were instructed to categorize the target words as quickly and accurately as they can, and ignore the image that appeared before them.

Implicit Association Test. In the IAT, participants categorize stimuli using two computer keys. In each trial, the target stimulus remained on screen until correct response. Participants had to fix incorrect responses with the correct response. The intertrial interval was $250 \mathrm{~ms}$. In the critical blocks, participants respond with the left key to stimuli of two categories (e.g., "Kevin" and "Good words"), and with the right key to stimuli of two other categories (e.g., "Brian" and "Bad words"). In two of these blocks, Kevin and Good words shared the same response key, and in the other two critical block, Brian and Good words shared the same response key. When "Kevin" and "Good words" shared the same key, people with more positive associations for Kevin than Brian should respond more quickly. Therefore, the difference between the average response latencies 
in blocks that assigned Kevin and Good words to the same key and blocks that assigned Brian and Good words to the same key is interpreted as automatic preference. The sevenblock IAT followed the procedure described in Nosek, Greenwald, and Banaji (2005). The IAT had 120 critical trials for scoring the Brian-Kevin preference score.

Sorting Paired Features Task. In the SPF, two pairs of category labels appear in each of the four corners of the screen. In each trial, participants sort pair stimuli (e.g., an image of Kevin and the word wonderful) to one of four category pairs (e.g., Kevin+pleasant, Kevin+unpleasant, Brian+pleasant, and Brian+unpleasant). Faster sorting indicates a stronger association between the pair categories. Therefore, a person who prefers Kevin over Brian will be faster on trials that present Kevin with pleasant words or Brian with unpleasant words than on trials that present Kevin with unpleasant words or Brian with pleasant words. We used three blocks of 40 trials. In each trial, the target stimulus appeared until correct response. Participants had to fix incorrect responses with the correct response. The intertrial interval was $250 \mathrm{~ms}$.

\section{Materials}

Target men. In Experiment 1, one photo of a White man was always Brian, and another photo of a White man was always Kevin (the photos were taken from Minear \& Park, 2004). In Experiments 2 and 4, to increase the stimulus sample, for each participant, two of 12 possible White man photos (from Payne, 2001) were randomly assigned to each man. In Experiment 3, the two images were selected from ten photos of Black men (from Gawronski, Cunningham, LeBel, \& Deutsch, 2010). For the indirect measures, we used five stimuli for each men: KEVIN, kevin [BRIAN, brian] and three 
image stimuli: the photo used in the induction, a vertical flip of the photo (mirror image), and a gray-scale version of the photo.

Evaluative stimuli. In Experiment 1-3, word selection was based on previous work on the Project Implicit website (Bar-Anan \& Nosek, 2014; Nosek et al., 2007). The EPT used the same words as the SPF (Pleasure, Love, Wonderful, Paradise, Cheer, Splendid, Bomb, Abuse, Sadness, Pain, Poison, Grief), with the addition of 16 words (Friend, Glee, Smile, Enjoy, Delight, Beautiful, Attractive, Likeable, Ugly, Dirty, Stimtmmnk, Noxious, Humiliate, Annoying, Disgusting, Offensive). The words used in IAT were Pleasure, Love, Wonderful, Laughter, Happy, Glorious, Joy, Peace, Awful, Failure, Agony, Hurt, Horrible, Terrible, Nasty, and Evil. In Experiment 4, the SPF, EPT, and the IAT all used the same words stimuli taken from Gawronski et al. (2015): pleasant, good, outstanding, beautiful, magnificent, marvelous, excellent, appealing, delightful, nice, unpleasant, bad, horrible, miserable, hideous, dreadful, painful, repulsive, awful, ugly. For the IAT, SPF, and EPT, the labels of the attribute categories were Pleasant words and Unpleasant words in Experiments 1-3, and Positive words and Negative words in Experiment 4.

Neutral stimuli. The Chinese pictographs in the AMP were retrieved from Keith Payne's website (http://bkpayne.web.unc.edu/research-materials/).

Behaviors. The behaviors were selected for each participant from a pool of 23 positive and 23 negative behaviors (see web supplement), collected from previous impression formation research (Bar-Anan \& Nosek, 2012; Ranganath \& Nosek, 2008). 


\section{Results}

\section{Data Processing}

For each measure, we computed a preference for Brian over Kevin.

EPT. Because there are many variations for computing EPT scores, we selected the scoring method that showed the best performance out of 1,536 scores created by enumerating a number of scoring parameters (Bar-Anan, 2016). We deleted error responses and replaced latencies longer than $800 \mathrm{~ms}$ with $800 \mathrm{~ms}$ (winsorizing). For each of the three blocks, we then subtracted the average log-transformed latencies of conditions compatible with preference for Brian (Brian-good; Kevin-bad) from the average log-transformed latencies of the conditions incompatible with that preference (Kevin-good; Brian-bad). We then divided each block's difference score by the standard deviation of the latency in that block. The preference score was the average of the standardized scores of the three blocks. Because this scoring has never been used before, we also report two scores that were used in the research from which we adapted the EPT procedures. Bar-Anan and Nosek (2014) used a similar scoring method, but instead of winsorizing slow responses they deleted trials with response latencies faster or slower more than 2 standard deviations from the average latency of the trial's prime-target condition. Gawronski et al. (2015) used raw latencies (rather than log-transformation), deleted error-trials and trials with response latencies longer than 1,500ms (no winsorizing), computed one score (rather than averaging across blocks), and did not divide it by standard deviation.

AMP. The proportion of pleasant judgments after Kevin were subtracted for the proportion of pleasant judgments after Brian. 
IAT. We used the D600 algorithm recommended by Greenwald et al. (2003). The overall IAT $D$ score was an average of two $D$ scores computed for each pair of critical blocks (blocks 3 versus 6 , and blocks 4 versus 7). The $D$ score for each pair of blocks was the difference between the average latency of each of the two opposing blocks (e.g., block 3 versus block 6) divided by the standard deviation of the trials in both blocks together. A positive $D$ score indicated faster responding on average when Brian was paired with good words and Kevin with bad words compared to the reverse, interpreted as a preference for Brian compared to Kevin. $D$ was calculated after removing response latencies under $400 \mathrm{~ms}$ or over $10000 \mathrm{~ms}$, and included all other trials. Latencies of error responses were replaced with the block mean of correct latencies plus $600 \mathrm{~ms}$.

SPF. We tested two scores for the SPF. One was recommended by Bar-Anan et al. (2009): A $D$ score for each of the four trial conditions was computed within each block as the difference between the average latency of the trial condition and the average latency in the whole block, divided by the overall standard deviation of that block. Then, single-attitude object scores were computed as the difference between the good and bad $D$ scores of each category (e.g., the difference between the D scores of Kevin-Good and Kevin-Bad). A preference score for each block was computed as the difference between the two single-category scores. The SPF preference score was the average of the preference scores of the three blocks. We also explored a modification of this scoring method, copying the same method but using log latencies instead of raw latencies and winsorizing of latencies faster than $300 \mathrm{~ms}$ or slower than $3,300 \mathrm{~ms}$. 
Self-report

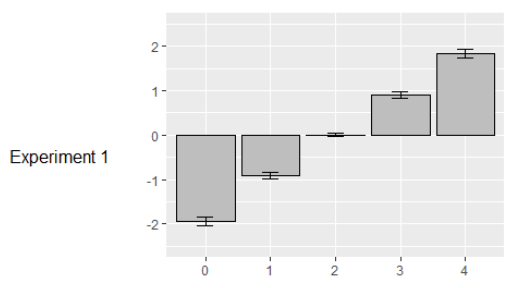

Experiment2
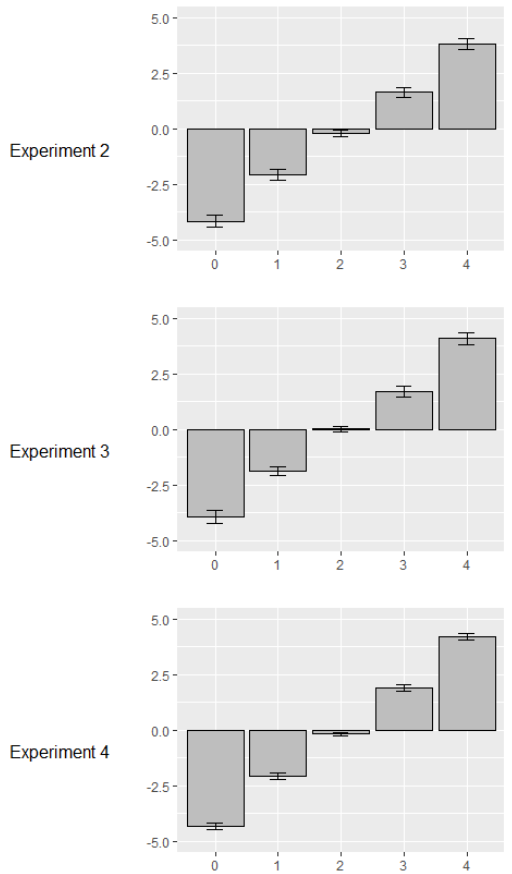

IAT
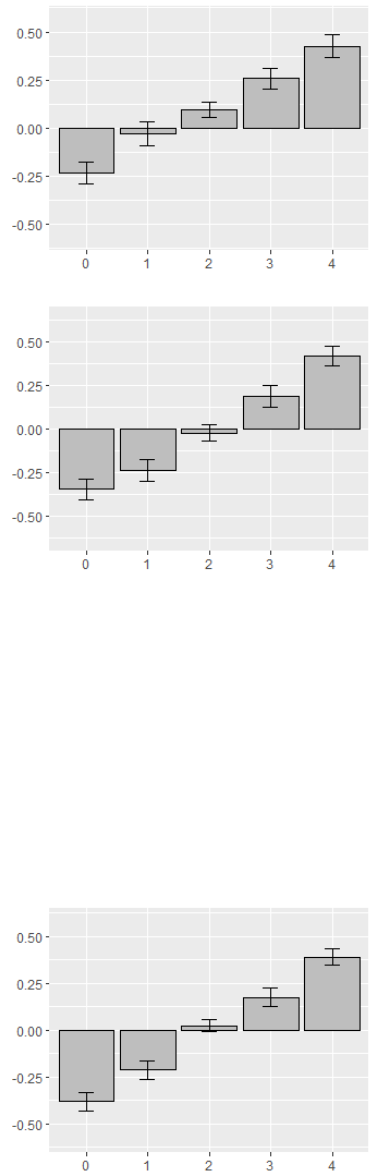

AMP
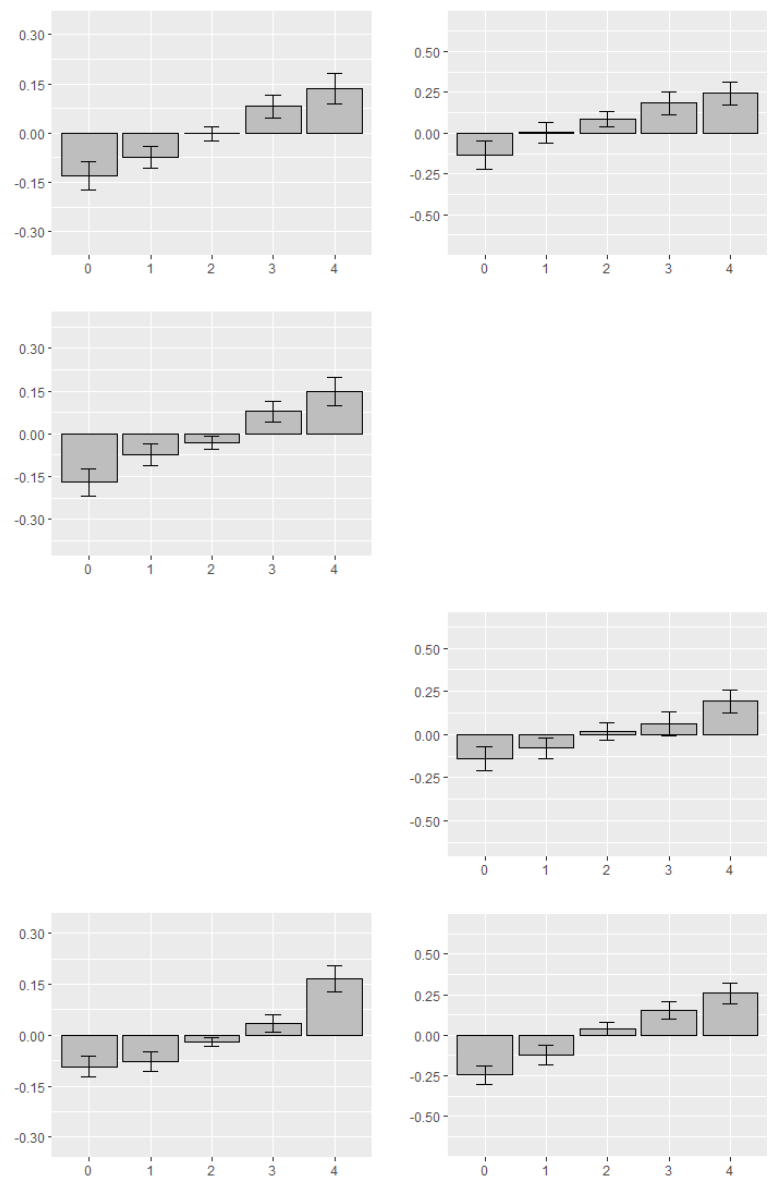

SPF

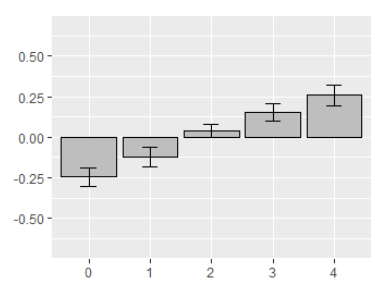

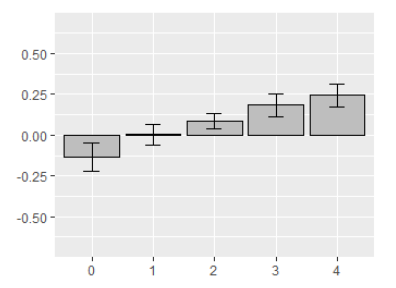

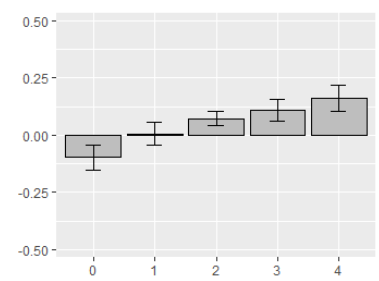

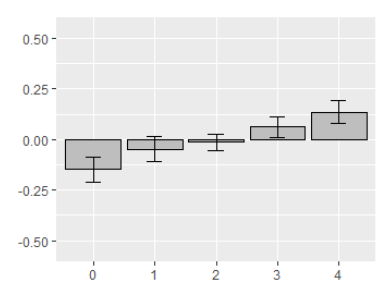

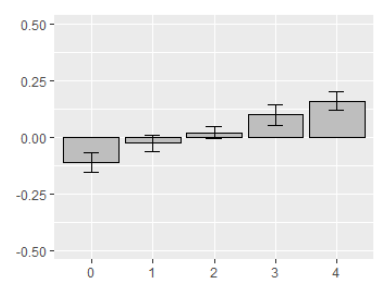

Figure 1. The effect of number of positive behaviors attributed to Brian (x-axis) on the preference score in each of the measures in each experiment; The self-report scores in Experiment 1 was standardized; For visual comparisons, the ranges of the y-axes for the indirect measures are always three pooled standards deviations; The range of the self-report graphs was six pooled standard deviations. Error-bars are 95\% confidence intervals. 
Direct evaluation of the behaviors. The preference score was the difference between the average rating of the behaviors performed by Kevin and the average rating of the behaviors performed by Brian.

Direct evaluation of the men. The preference score was the difference between Kevin's average trait rating and Brian's average trait rating. In Experiment 1, the ratings were standardized within each question format condition because response values on the VAS ranged from -100 to 100 .

\section{Behavior Evaluation}

In Experiments 1-3, all the positive behaviors were rated as more positive than all the negative behaviors, with a minimum effect size of $d s=1.80,1.68,2.01$.

\section{Sensitivity to Evaluative Information}

Figure 1 presents the average preference as a function of number of positive behaviors attributed to Brian, for each measure in each experiment. In Experiment 1, the measures were slightly biased by pre-existing preference for the combination of the name 'Brian' and the image selected for Brian over 'Kevin' and his image. In the other experiments, because the face images were selected randomly from a pull of images, preexisting attitudes did not influence the overall means.

Table 1 presents the internal consistencies of the measures and their intercorrelations. The measure of sensitivity to the induction was the correlation between each measure's score and the number of positive behaviors performed by Brian (Induction Condition in Table 1). Higher correlations indicate strong sensitivity to the evaluative information. For statistical inference from these results, we compared the Pearson correlations (Table 1). The IAT outperformed all the other measures in each experiment. The statistics for the comparison of the correlations of the induction condition with the IAT versus with the second best measure (AMP, AMP, SPF) were $Z s=2.73,4.01,5.84$, $p \mathrm{~s}=.006, .0001,<.0001 \mathrm{in}$ Experiments 1, 2, and 4, respectively. 
Table 1

The correlations of the evaluation measures with evaluative information and self-report

$\begin{array}{llllllll}\text { IAT } & \text { AMP } & \text { SPF } & \text { SPF }_{w} & \text { EPT } & \text { EPT }_{B N} & \text { EPT }_{G} & \text { Explicit }\end{array}$

\section{Experiment 1}

\begin{tabular}{lcccccccc}
$\mathbf{N}$ & 1,036 & 898 & 963 & 963 & 992 & 1,008 & 1,010 & 3,902 \\
Internal consistency & $.71_{\mathrm{c}}$ & $.85_{\mathrm{b}}$ & $.41_{\mathrm{d}}$ & $.48_{\mathrm{d}}$ & $.29_{\mathrm{e}}$ & $.33_{\mathrm{e}}$ & $.19_{\mathrm{f}}$ & $.91_{\mathrm{a}}$ \\
Induction condition & $.467_{\mathrm{b}}$ & $.364_{\mathrm{c}}$ & $.248_{\mathrm{d}}$ & $.268_{\mathrm{d}}$ & $.233_{\mathrm{de}}$ & $.219_{\mathrm{de}}$ & $.181_{\mathrm{e}}$ & $.756_{\mathrm{a}}$ \\
Explicit preference & $.525_{\mathrm{a}}$ & $.449_{\mathrm{b}}$ & $.251_{\mathrm{c}}$ & $.281_{\mathrm{c}}$ & $.279_{\mathrm{cd}}$ & $.253_{\mathrm{cd}}$ & $.188_{\mathrm{d}}$ & - \\
\hline Experiment 2 & & & & & & & & 1,980 \\
$\mathbf{N}$ & 1,070 & 947 & - & & - & & $.93_{\mathrm{a}}$ \\
Internal consistency & $.78_{\mathrm{c}}$ & $.87_{\mathrm{b}}$ & - & & - & & $.762_{\mathrm{a}}$ \\
Induction condition & $.517_{\mathrm{b}}$ & $.373_{\mathrm{c}}$ & - & & - & & -
\end{tabular}

\section{Experiment 3}

$\mathbf{N}$

Internal consistency

Induction condition

Explicit preference
927

.33

$.234_{\mathrm{bc}}$

$.251_{\mathrm{ab}}$
925

$.46 \mathrm{~b}$

$.285_{b}$

$.293_{\mathrm{a}}$
933

$.12_{\mathrm{d}}$

$.235_{\mathrm{bc}}$

$.263_{\mathrm{ab}}$
983

985

1,898

$.32 \mathrm{c}$

$.07_{\mathrm{d}}$

$.94 \mathrm{a}$

$.192_{\mathrm{c}}$

$.176_{\mathrm{c}}$

$.773_{\mathrm{a}}$

$.236_{\mathrm{ab}} \quad .208_{\mathrm{b}}$

\section{Experiment 4}

$\mathbf{N}$

1,446

1,417

1,415

1,518

1,563

1,567

6,063

Internal consistency

$.75_{\mathrm{c}} \quad .84_{\mathrm{b}}$

$.47 \mathrm{e}$

$.57 \mathrm{~d}$

$.29_{\mathrm{f}}$

$.43 \mathrm{e}$

$.25_{\mathrm{f}}$

$.93 \mathrm{a}$

Induction condition $\quad .540 \mathrm{~b}$

$.349_{\mathrm{c}} \quad .337_{\mathrm{c}}$

$.373_{\mathrm{c}}$

$.257_{\mathrm{d}}$

$.231_{\mathrm{de}} \quad .172_{\mathrm{e}}$

$.790_{\mathrm{a}}$

Explicit preference

$.564 \mathrm{a}$

$.449_{b}$

$.375_{\mathrm{c}}$

$.421_{\mathrm{bc}} \quad .280_{\mathrm{d}}$

$.257_{\mathrm{d}}$

$.192_{\mathrm{e}}$

Avg. with induction $\quad .508$

.362

.274

.309

.242

.214

.176

.770

$\mathbf{N}$ for $\mathbf{8 0 \%}$ power 28

57

101

79

131

168

250

10

$\mathbf{N}$ for $\mathbf{9 9 \%}$ power

61

130

242
583

20

Notes. $S P F_{w}$ is the score based on winsorized latencies. EPT $T_{B N}$ is the EPT score recommended by Bar-Anan and Nosek (2014); EPT ${ }_{G}$ is the score adapted from Gawronski et al. (2015). All the correlations are significantly different from zero; In each row, a different subscript indicates a significant difference ( $p<.05$, two-sided), computed following instructions from Zou (2007) for correlations and from Feldt (1969) for internal consistency; Internal consistency was computed from three parcels; Power to detect each correlation was computed for the average correlations, with $p=.05$, two-tailed. 
The AMP was significantly more sensitive than the SPF in Experiment $1, Z=$ $2.29, p=.021$, but numerically less sensitive in Experiment $4, Z=0.92, p=.358$. We speculate that the SPF improved because of the word stimuli used in Experiment 4 (the IAT and the EPT also showed better sensitivity using those words). Although the SPF showed numerically stronger correlations than the EPT in Experiments 1, 3, and 4, the differences reached statistical significance only in Experiment $4, Z=3.49, p=.0005$, and not in Experiments 1 and 3, Zs $=0.82,1.15, p s=.409, .248$. In the web supplement, we report similar results with two alternative tests for significant differences: one based on Spearman correlations and the other based on multiple regressions.

\section{Predicting induction condition above self-reported preference}

We explored whether each of the indirect measures shared any variance with the induction condition that was not shared with the self-report. Table 2 shows the results of two analysis strategies. One was a multiple regression predicting the induction condition (number of positive behavior attributed to Brian) from the indirect measure and the selfreport measure. The regression tested whether the indirect measure added any explained variance to the model. Another analysis was the same multiple regression computed as a structural equation model (SEM), specifying measurement models for each predictor, to predict the outcome variable from the latent variables, thus clearing the error-variance associated with each factor (Westfall \& Yarkoni, 2016). This analysis tested whether the explained variance gained by adding the indirect measure to the model can be attributed to a theoretical construct different than the construct measured with the self-report measure (as opposed to attributing the gain to limits in the reliability of the self-report measure). Based on previous research and theory pertaining to the effects of simple evaluative information, we expected no difference in the effect of the present manipulation on the two constructs. However, because the reliability of the self-report was high but not perfect, it was reasonable to assume that highly reliable indirect measure could improve the prediction in the simple multiple regression analysis.

The IAT had the best performance, significantly improving prediction in all three experiments. In two of the three experiments, the IAT's shared variance with the selfreport remained significant even after removing error-variance with SEM. The SPF always showed significant improvement of the prediction in all three experiments. With 
SEM, the SPF's improvement remained significant in only one of the three experiments. There was no statistically significant evidence that the EPT and the AMP improved the prediction in any of the experiments.

Table 2

Standardized parameter estimates from simultaneous prediction of the induction condition from direct and indirect measures (in parentheses: p-value)

\begin{tabular}{clllll}
\hline \multirow{2}{*}{ Experiment } & Indirect & Indirect & Direct & Indirect & Direct \\
& Measure & Measure & Measure & Measure & Measure \\
1 & IAT & $.096(<.0001)$ & $.707(<.0001)$ & $.063(.073)$ & $.751(<.0001)$ \\
1 & AMP & $.019(.413)$ & $.767(<.0001)$ & $-.021(.442)$ & $.818(<.0001)$ \\
1 & SPF & $.057(.009)$ & $.742(<.0001)$ & $.076(.053)$ & $.754(<.0001)$ \\
1 & EPT & $.023(.296)$ & $.732(<.0001)$ & $.022(.320)$ & $.760(<.0001)$ \\
\hline 2 & IAT & $.124(<.0001)$ & $.690(<.0001)$ & $.112(<.0001)$ & $.713(<.0001)$ \\
2 & AMP & $.020(.410)$ & $.751(<.0001)$ & $-.011(.687)$ & $.789(<.0001)$ \\
\hline 3 & SPF & $.068(.002)$ & $.761(<.0001)$ &. $\mathbf{0 9 3}(.014)$ & $.743(<.0001)$ \\
3 & EPT & $.031(.148)$ & $.757(<.0001)$ & $.023(.258)$ & $.802(<.0001)$ \\
\hline 4 & IAT &. $\mathbf{1 4 0}(<.0001)$ & $.698(<.0001)$ & $.135(<.0001)$ & $.722(<.0001)$ \\
4 & AMP & $-.006(.740)$ & $.792(<.0001)$ & $-.038(.071)$ & $.831(<.0001)$ \\
4 & SPF & $.046(.009)$ & $.788(<.0001)$ & $.032(.262)$ & $.799(<.0001)$ \\
4 & EPT & $.039(.018)$ & $.783(<.0001)$ & $.008(.634)$ & $.811(<.0001)$
\end{tabular}

Notes. Each multiple regression predicted the induction condition from the indirect measure and the self-report measure; The SEM analyses used three indicators for each predictor - three parcels for each indirect measure, and the self-reported preferences on the three traits (trustworthiness, likeability, friendliness). The SEM analysis was conducted with R's package lavaan (version 0.5-20, Rosseel, 2012); In bold: significant prediction for the indirect measure. 


\section{General Discussion}

Four experiments compared the sensitivity of four indirect evaluation measures to clear explicit evaluative information. The results revealed that the IAT was the most sensitive. The second most sensitive measure was the AMP because, across experiments, it showed stronger average sensitivity than the SPF. However, it was not superior to the SPF in Experiment 4, so its advantage over the SPF is less certain. EPT was always inferior to the IAT and the AMP, but the SPF was significantly stronger than the EPT only in Experiment $4^{3}$. The superiority of the IAT and the inferiority of the EPT are mostly compatible with the evidence collected earlier in comparative and noncomparative studies using those measures. The SPF performed slightly better than in the past, especially in Experiment 4, whereas the AMP performed somewhat worse, especially in Experiment 4.

All studied indirect measures were sensitive to evaluative information, but the measures differed substantially in the sample sizes required for reliably detecting effects. Table 1 provides post-hoc computations of sample-size required to achieve $80 \%$ and $99 \%$ power to detect a statistically reliable effect (a correlation with the induction) for each measure. For equal probability to detect the same effect the measures require substantially different numbers of participants. Taking $99 \%$ power, for example, the selfreport measure requires just 20 participants, whereas the indirect measures require, 61 for the IAT, 130 for the AMP, 182 for SPF, and 242 for EPT. These dramatic differences have significant implications for research efficiency. If generalizable, using EPT requires $300 \%$ more participants than the IAT to conduct equivalently high-powered research in this context.

As Table 1 shows, the SPF and the EPT performed much better when we used scoring methods that we identified as high performing approaches, but are different than those used in previous investigations. Particularly, it seems that winsorizing slow responses improves performance in both cases. In future work, we will investigate that possibility with a wider sample of populations, settings, stimuli, study designs, and

\footnotetext{
${ }^{3}$ In a correlational comparative study, Bar-Anan and Nosek (2014) found that the Brief-IAT (BIAT; Sriram \& Greenwald, 2009) - a recent modification of the IAT-showed psychometric qualities comparable or better than the IAT. An unpublished experiment (Bar-Anan \& Nosek, 2016) found that the BIAT is more sensitive than the AMP to direct evaluative information.
} 
attitude domains. For now, we recommend researchers to explore winsorizing-based scores, when using the SPF and the EPT (see Richetin, Costantini, Perugini, \& Schönbrodt, 2015, for initial findings that winsorizing might also be useful for scoring the IAT).

The IAT and the SPF had unique variance with the evaluative information above and beyond the variance captured by the self-report measure. The SEM analyses found some evidence for divergent validity, suggesting that evaluative information has unique influence on constructs captured by the IAT and the SPF, not captured by the selfreported evaluation. This might suggest that even straightforward evaluative information causes some discrepancy between deliberate and automatic evaluation. As a speculation, perhaps automatic evaluation is more sensitive than deliberate evaluation to simple statistical contingencies, and less sensitive to information integration processes (Ratliff \& Nosek, 2010; Rydell et. al, 2007). The fact that the AMP and the EPT did not capture that unique variance might suggest that the IAT and SPF are more suitable for studying discrepancies between automatic and deliberate evaluation (Bar-Anan \& Nosek, 2012). However, it is premature to conclude that that unique variance is evaluative. Perhaps the IAT and the SPF showed sensitivity to non-attitudinal effects of the induction procedure.

In the present research, one possible reason for the superiority of the IAT and the relatively good performance of the SPF is that these two measures are designed specifically for the measurement of preferences between two attitude objects. The AMP and the EPT have the advantage of being able to measure evaluations of more than two objects. There is currently only initial evidence that the SPF (Bar-Anan et al., 2009) or IAT variants (Axt, Ebersole, \& Nosek, 2014) can provide valid measures for more than two objects. Further research is needed for comparing the quality of indirect measures in capturing attitudes toward more than two objects.

The present research focused on sensitivity to evaluation. There are other important considerations in the selection of indirect measures that the present research has not addressed. Perhaps the most important features are the ability to capture automatic evaluation, predict behavior, and overcome faking efforts. We are unaware of any research that directly compared the sensitivity of the measures we tested to faking efforts. In the only known comparative faking study, participants were more successful in 
faking the Single-Target IAT (Karpinski \& Steinman, 2006) than the IAT (Stieger, Göritz, Hergovich, \& Voracek, 2011). Research that compared different indirect measures on predicting automatic and non-automatic behavior is scarce and has always suffered from small sample sizes that prevented strong conclusions (e.g., Payne et al., 2008; Spruyt et al., 2007). So far, we have not been able to conduct such a study because we have not found any behavioral measure that consistently shows stronger relations with automatic evaluation than with deliberate evaluation.

\section{Summary}

For investigating sensitivity to experimentally formed attitudes, we found clear evidence that the IAT is more sensitive than the AMP, SPF, and EPT, and relatively clear evidence that the AMP is more sensitive than the EPT. Power estimates suggest that the measures differ substantially in sensitivity and research efficiency. Because these measures are important tools in investigating attitudes and automatic evaluation, further comparative studies about the relative psychometric qualities of these measures and about their sensitivity to various forms of evaluative information are essential. 


\section{References}

Ajzen, I., \& Fishbein, M. (1980). Understanding attitudes and predicting social behavior. Englewood Cliffs, NJ: Prentice-Hall.

Albarracin, D., Johnson, B. T., \& Zanna, M. P. (Eds.). (2014). The handbook of attitudes. Psychology Press.

Anderson, N. H. (1965). Averaging versus adding as a stimulus-combination rule in impression formation. Journal of Experimental Psychology, 70(4), 394-400.

Asch, S. E. (1946). Forming impressions of personality. The Journal of Abnormal and Social Psychology, 41(3), 258-290.

Axt, J. R., Ebersole, C. R. \& Nosek, B. A. (2014). The rules of implicit evaluation by race, religion, and age. Psychological Science, 25, 1804-1815.

Banse, R., Seise, J., \& Zerbes, N. (2001). Implicit attitudes towards homosexuality: Reliability, validity, and controllability of the IAT. Zeitschrift für experimentelle Psychologie, 48(2), 145-160.

Bar-Anan, Y. (2010). Strategic modification of the evaluative priming effect does not reduce its sensitivity to uncontrolled evaluations. Journal of Experimental Social Psychology, 46(6), 1101-1104.

Bar-Anan, Y. (2016). Exploring Scoring Algorithms for the Evaluative Priming in Evaluative Learning Experiments. Unpublished Manuscript. https://osf.io/7yzzu/

Bar-Anan, Y., \& Nosek, B. A. (2012). Reporting intentional rating of the primes predicts priming effects in the affective misattribution procedure. Personality and Social Psychology Bulletin, 38(9), 1194-1208.

Bar-Anan, Y., \& Nosek, B. A. (2013). The effect of number of trials and stimulus set on the psychometric qualities of the affective misattribution procedure. Unpublished Manuscript. https://osf.io/dspar/.

Bar-Anan, Y., \& Nosek, B. A. (2014). A comparative investigation of seven indirect attitude measures. Behavior research methods, 46(3), 668-688.

Bar-Anan, Y., \& Nosek, B. A. (2016). An Experimental Comparison of the Affect Misattribution Procedure and the Brief Implicit Association Test. Unpublished manuscript. https://osf.io/4yn3r/ 
Bar-Anan, Y., Nosek, B. A., \& Vianello, M. (2008). A comparison between the SPF and evaluative priming. Unpublished manuscript. http://www.briannosek.com/spf/.

Bar-Anan, Y., Nosek, B. A., \& Vianello, M. (2009). The sorting paired features task: A measure of association strengths. Experimental Psychology, 56, 329-343.

Blaison, C., Imhoff, R., Hühnel, I., Hess, U., \& Banse, R. (2012). The affect misattribution procedure: Hot or not?. Emotion, 12(2), 403.

Bosson, J. K., Swann, W. B., \& Pennebaker, J. W. (2000). Stalking the perfect measure of implicit self-esteem: The blind men and the elephant revisited? Journal of Personality and Social Psychology, 79, 631-643.

Conrey, F. R., Sherman, J. W., Gawronski, B., Hugenberg, K., \& Groom, C. J. (2005). Separating multiple processes in implicit social cognition: the quad model of implicit task performance. Journal of personality and social psychology, 89(4), 469.

Cunningham, W. A., Preacher, K. J., \& Banaji, M. R. (2001). Implicit attitude measures: Consistency, stability, and convergent validity. Psychological science, 12(2), 163-170.

De Houwer, J. (2006). What are implicit measures and why are we using them. The handbook of implicit cognition and addiction, 11-28.

De Houwer, J., \& Bruycker, E. (2007). The implicit association test outperforms the extrinsic affective Simon task as an implicit measure of inter-individual differences in attitudes. British Journal of Social Psychology, 46(2), 401-421.

Egloff, B., \& Schmukle, S. C. (2002). Predictive validity of an Implicit Association Test for assessing anxiety. Journal of personality and social psychology, 83(6), 1441.

Fazio, R. H., \& Olson, M. A. (2003). Implicit measures in social cognition research: Their meaning and use. Annual review of psychology, 54(1), 297-327.

Fazio, R. H., Sanbonmatsu, D. M., Powell, M. C., \& Kardes, F. R. (1986). On the automatic activation of attitudes. Journal of Personality and Social Psychology, 50, $229-238$.

Feldt, L. S. (1969). A test of the hypothesis that cronbach's alpha or kuder-richardson coefficent twenty is the same for two tests. Psychometrika, 34(3), 363-373.

Gawronski, B., Deutsch, R., LeBel, E. P., \& Peters, K. R. (2008). Response interference as a mechanism underlying implicit measures: Some traps and gaps in the assessment of 
mental associations with experimental paradigms. European Journal of Psychological Assessment, 24(4), 218-225.

Gawronski, B., Cunningham, W. A., LeBel, E. P., \& Deutsch, R. (2010). Attentional influences on affective priming: Does categorisation influence spontaneous evaluations of multiply categorisable objects?. Cognition and Emotion, 24(6), 1008-1025.

Gawronski, B., Gast, A., \& De Houwer, J. (2015). Is evaluative conditioning really resistant to extinction? Evidence for changes in evaluative judgements without changes in evaluative representations. Cognition and Emotion, 29(5), 816-830.

Gregg, A. P., Seibt, B., \& Banaji, M. R. (2006). Easier done than undone: Asymmetry in the malleability of implicit preferences. Journal of Personality and Social Psychology, 90(1), 1-20

Greenwald, A. G., McGhee, D. E., \& Schwartz, J. K. L. (1998). Measuring individual differences in implicit cognition: The Implicit Association Test. Journal of Personality and Social Psychology, 74, 1464-1480.

Greenwald, A. G., Nosek, B. A., \& Banaji, M. R. (2003). Understanding and using the Implicit Association Test: I. An improved scoring algorithm. Journal of Personality and Social Psychology, 85(2), 197-216.

Horcajo, J., Briñol, P., \& Petty, R. E. (2010). Consumer persuasion: Indirect change and implicit balance. Psychology \& Marketing, 27(10), 938-963.

Hovland, C. I., Janis, I. L., \& Kelley, H. H. (1953). Communication and Persuasion. New Haven, CT: Yale University Press.

Jones, E. E., \& Sigall, H. (1971). The bogus pipeline: a new paradigm for measuring affect and attitude. Psychological Bulletin, 76(5), 349.

Karpinski, A., \& Steinman, R. B. (2006). The Single Category Implicit Association Test as a measure of implicit social cognition. Journal of Personality and Social Psychology, 91, 16-32.

Klauer, K. C., \& Musch, J. (2003). Affective priming: Findings and theories. The psychology of evaluation: Affective processes in cognition and emotion, 7-49.

Krause, S., Back, M. D., Egloff, B., \& Schmukle, S. C. (2011). Reliability of implicit self-esteem measures revisited. European Journal of Personality,25(3), 239-251. 
Leary, M. R., \& Kowalski, R. M. (1990). Impression management: A literature review and two-component model. Psychological bulletin, 107(1), 34.

McGaugh, J. L. (2004). The amygdala modulates the consolidation of memories of emotionally arousing experiences. Annu. Rev. Neurosci., 27, 1-28.

Minear, M., \& Park, D. C. (2004). A lifespan database of adult facial stimuli. Behavior Research Methods, Instruments, \& Computers, 36(4), 630-633.

Moran, T., Bar-Anan, Y., \& Nosek, B. A. (in press). The effect of the validity of cooccurrence on automatic and deliberate evaluation. European Journal of Social Psychology.

Nosek, B. A. (2005). Moderators of the relationship between implicit and explicit evaluation. Journal of Experimental Psychology: General, 134, 565-584.

Nosek, B. A., Greenwald, A. G., \& Banaji, M. R. (2007). The Implicit Association Test at age 7: A methodological and conceptual review. In J. A. Bargh (Ed.), Social Psychology and the Unconscious: The Automaticity of Higher Mental Processes (pp. 265-292). New York: Psychology Press.

Nosek, B. A., \& Greenwald, A.G. (2009). (Part of) the case for a pragmatic approach to validity: Comment on De Houwer, Teige-Mocigemba, Spruyt, and Moors (2009). Psychological Bulletin, 135, 373-376.

Nosek, B. A., Greenwald, A. G., \& Banaji, M. R. (2005). Understanding and using the Implicit Association Test: II. Method variables and construct validity. Personality and Social Psychology Bulletin, 31(2), 166-180.

Nosek, B. A., Hawkins, C. B., \& Frazier, R. S. (2011). Implicit social cognition: From measures to mechanisms. Trends in cognitive sciences,15(4), 152-159.

Nosek, B. A., Hawkins, C. B., \& Frazier, R. S. (2012). Implicit social cognition. Handbook of social cognition, 31-53.

Olson, M. A., \& Fazio, R. H. (2001). Implicit attitude formation through classical conditioning. Psychological Science, 12(5), 413-417.

Payne, B. K. (2001). Prejudice and perception: the role of automatic and controlled processes in misperceiving a weapon. Journal of personality and social psychology, 81(2), 181. 
Payne, B. K., Cheng, C. M., Govorun, O., \& Stewart, B. D. (2005). An inkblot for attitudes: Affect misattribution as indirect measurement. Journal of Personality and Social Psychology, 89, 277-293.

Payne, B. K., Govorun, O., \& Arbukle, N. L. (2008). Automatic attitudes and alcohol: Does implicit liking predict drinking? Cognition and Emotion, 22, 238-271.

Payne, B. K., \& Lundberg, K. (2014). The Affect Misattribution Procedure: Ten Years of Evidence on Reliability, Validity, and Mechanisms. Social and Personality Psychology Compass, 8(12), 672-686.

Peters, K. R., \& Gawronski, B. (2011). Are We Puppets on a String? Comparing the Impact of Contingency and Validity on Implicit and Explicit Evaluations. Personality and Social Psychology Bulletin, 37(4), 557-569.

Petty, R. E., \& Cacioppo, J. T. (1986). The elaboration likelihood model of persuasion. Advances in experimental social psychology, 19, 123-205

Ranganath, K. A., \& Nosek, B. A. (2008). Implicit attitude generalization occurs immediately; explicit attitude generalization takes time. Psychological Science, 19(3), 249-254.

Ranganath, K. A., Smith, C. T., \& Nosek, B. A. (2008). Distinguishing automatic and controlled components of attitudes from direct and indirect measurement methods. Journal of Experimental Social Psychology, 44, 386-396.

Ratliff, K. A., \& Nosek, B. A. (2010). Creating distinct implicit and explicit attitudes with an illusory correlation paradigm. Journal of Experimental Social Psychology, 46(5), 721-728.

Richetin, J., Costantini, G., Perugini, M., \& Schönbrodt, F. (2015). Should we stop looking for a better scoring algorithm for handling Implicit Association Test data? Test of the role of errors, extreme latencies treatment, scoring formula, and practice trials on reliability and validity. PloS one, 10(6), e0129601.

Rosseel, Y. (2012). lavaan: An R package for structural equation modeling. Journal of Statistical Software, 48(2), 1-36.

Rudolph, A., Schröder-Abé, M., Schütz, A., Gregg, A. P., \& Sedikides, C. (2008).

Through a glass, less darkly? Reassessing convergent and discriminant validity in 
measures of implicit self-esteem. European Journal of Psychological Assessment, 24(4), 273-281.

Rydell, R. J., McConnell, A. R., Strain, L. M., Claypool, H. M., \& Hugenberg, K. (2007). Implicit and explicit attitudes respond differently to increasing amounts of counterattitudinal information. European Journal of Social Psychology, 37(5), 867-878.

Smith, C. T., De Houwer, J., \& Nosek, B. A. (2013). Consider the Source Persuasion of Implicit Evaluations Is Moderated by Source Credibility. Personality and Social Psychology Bulletin, 39(2), 193-205.

Scherer, L. D., \& Lambert, A. J. (2009). Contrast effects in priming paradigms: Implications for theory and research on implicit attitudes. Journal of personality and social psychology, 97(3), 383.

Scherer, L. D., \& Lambert, A. J. (2012). Implicit race bias revisited: On the utility of task context in assessing implicit attitude strength. Journal of Experimental Social Psychology, 48(1), 366-370.

Spruyt, A., Hermans, D., De Houwer, J., Vandekerckhove, J., \& Eelen, P. (2007). On the predictive validity of indirect attitude measures: Prediction of consumer choice behavior on the basis of affective priming in the picture-picture naming task. Journal of Experimental Social Psychology, 43(4), 599-610.

Sriram, N., \& Greenwald, A. G. (2009). The brief implicit association test. Experimental Psychology, 56, 283-294.

Stahl, C., \& Unkelbach, C. (2009). Evaluative learning with single versus multiple unconditioned stimuli: the role of contingency awareness. Journal of Experimental Psychology: Animal Behavior Processes, 35(2), 286.

Stickgold, R., \& Walker, M. P. (2005). Memory consolidation and reconsolidation: what is the role of sleep?. Trends in neurosciences, 28(8), 408-415.

Stieger, S., Göritz, A.S., Hergovich, A., Voracek, M., 2011. Intentional faking of the Single Categorie Implicit Association Test and the Implicit Association Test. Psychological Report, 109, 219-230.

Walther, E., \& Langer, T. (2008). Attitude formation and change through association: An evaluative conditioning account. Attitudes and attitude change, 87-109. 
Westfall, J., \& Yarkoni, T. (2016). Statistically controlling for confounding constructs is harder than you think. PloS one, 11(3), e0152719.

Zlotnick, E., Dzik, A., Bar-Anan, Y. (2015). PIPE (Version 0.3) [Computer software].

Zou, G.Y. (2007). Toward using confidence interval to compare correlations.

Psychological Methods, 12, 399-413. 